\title{
Behavior of UDP Traffic over LTE Network
}

\author{
Tauseef Ahmad \\ ZHCET, A.M.U. \\ Aligarh, U.P., \\ India- 202002
}

\begin{abstract}
Modern mobile communication networks provide a variety of voice and data services. The latest set of mobile technology specifications by the 3rd Generation Partnership Project (3GPP) is referred to as Long Term Evolution (LTE). In this paper, the performance of a wireless network where LTE is used at the Medium Access Control (MAC) layer is evaluated. User Datagram Protocol (UDP) traffic is considered for the evaluation. The major performance parameters that are focused are average throughput, end-to-end delay, and jitter. Experiments are performed to evaluate the effect for varying bandwidth, number of subscribers, and packet size on these performance parameters for downlink scenarios.
\end{abstract}

\section{General Terms}

Wireless mobile communication, long term evolution.

\section{Keywords}

eNodeB, Jitter, LTE, Latency, MAC, Performance Evaluation, Throughput, 3GPP.

\section{INTRODUCTION}

The major wireless communication standards evolutions are: It starts from AMPS and moves up to LTE-A. The first wireless evolution standard was called Advanced Mobile Phone System (AMPS), consist of analogue system. Later, in the early 1990s Global System for Mobile Communication (GSM) came into existence and was considered as first digital communication system and it is also called $2 \mathrm{G}$ standards. Then in mid 90s, 2.5G came into existence which is called General Packet Radio Service (GPRS), it supports data bit rate up to $114 \mathrm{Kbps}$. In the end of 90s Enhanced Data rate for GSM evolution (EDGE) came into existence and it was considered as the key for beginning of the 3G. In 2002 Universal Mobile Telecommunication System (UMTS) came into existence, which is considered as complete $3 \mathrm{G}$ standards. HSPA is considered as the starting of migration of $3 \mathrm{G}$ to $4 \mathrm{G}$ in the beginning of 21th century. HSPA has improved the data bit rate to reach up to $14 \mathrm{Mbps}$ and is known as 3.5 Generation. Therefore, LTE is the major key which leads the communication technology to start the 4th Generation level.

There has been a rapid increase in the use of data carried by cellular services and the accelerating increase has been termed the "data explosion" .To overcome this and to meet the increased demands of data transmission speeds and lower latency, a development of cellular technology is required[1].

The UMTS cellular technology has upgraded the dubbed version of LTE. The idea is that LTE will enable to access higher speed along with much lower packet latency (a growing requirement for many services these days). Additionally, 3GPP LTE will enable cellular communications services to move forward to meet the needs for cellular technology by 2017 and much beyond.

\subsection{G LTE Evolution}

There are lot of technical changes between LTE and its predecessors, but it is nevertheless considered as the evolution of its predecessors i.e. 3G standards. There are lots of similarities between LTE and its predecessors in terms of their architecture, but it has major change in form of radio interface, i.e. OFDMA / SC-FDMA instead of CDMA.

To differentiate between LTE and other cellular technologies, it is better to take a look on the specification of LTE and its predecessors. It will give a brief idea about the major difference between them. LTE can provide a further evolution of functionality, increased speeds and general improved performance [3].

Table 1 shows comparison of LTE vs. other previous technologies.

Table 1: LTE Vs Other Technologies

\begin{tabular}{|c|c|c|c|c|}
\hline & $\begin{array}{c}\text { WCDMA } \\
\text { (UMTS) }\end{array}$ & $\begin{array}{c}\text { HSPA } \\
\text { HSDPA } \\
\text { HSUPA }\end{array}$ & HSPA+ & LTE \\
\hline $\begin{array}{c}\text { Max. } \\
\text { Downlink } \\
\text { Speed (bps) }\end{array}$ & $384 \mathrm{~K}$ & $14 \mathrm{M}$ & $28 \mathrm{M}$ & $100 \mathrm{M}$ \\
\hline $\begin{array}{c}\text { Max. } \\
\text { Uplink } \\
\text { Speed (bps) }\end{array}$ & $128 \mathrm{~K}$ & $5.7 \mathrm{M}$ & $11 \mathrm{M}$ & $50 \mathrm{M}$ \\
\hline $\begin{array}{c}\text { Latency } \\
\text { 3GPP } \\
\text { Release }\end{array}$ & $150 \mathrm{~ms}$ & $100 \mathrm{~ms}$ & $50 \mathrm{~ms}$ & $\sim 10 \mathrm{~ms}$ \\
\hline $\begin{array}{c}\text { Access } \\
\text { Methodolo- } \\
\text { gy }\end{array}$ & CDMA & Rel $5 / 6$ & Rel 7 & Rel 8 \\
\hline
\end{tabular}

In this paper, we evaluated the performance of a wireless network where LTE is used at the Medium Access Control (MAC) layer. We have considered User Datagram Protocol (UDP) traffic. The major performance parameters that we focused on are average throughput, end-to-end delay, and jitter. We have evaluated the effect of varying bandwidth, number of subscribers, and packet size on these performance parameters for downlink scenarios.

Rest of the paper is organized as follows. In Section 2 we discuss LTE architecture. Section 3 describes the network model and implementation. In Section 4 we discuss the results and evaluate its performance by measuring the performance metrics, e.g., average end-to-end delay, average jitter, and average throughput. Finally, we conclude our work in Section 5 . 


\section{LONG TERM EVOLUTION/SYSTEM ARCHITECTURE EVOLUTION (LTE/SAE)}

LTE along with SAE provides the improvement in the network architecture of LTE. The major improvement is that it decreases the total number of nodes in core network and it causes decrease in the complexity of the LTE architecture. Therefore, it is very convincing for the operators that are using HSPA to upgrade to LTE networks. Another major change is; in LTE the network becomes flatter. Flatter network means that the stations communicate with each other without any mediators for example routers. Due to its flatter characteristics, total time taken for each packet to travel becomes lesser as compared to previous technologies, and overall latency gets improved. In LTE, some nodes work collectively and are merged or divided with each other since LTE/SAE elements have the ability to take place and substitute the user and/or control nodes. We can say as example, the Radio Network Controller (RNC) in previous technology is split in two parts i.e. Access Gate Way (AGW) and eNodeB. On the other hand, the Core Network elements of previous technology such as SGSN (Serving GPRS Support Node) and GGSN (Gateway GPRS Support Node) or PDSN (Packet Data Serving Node) are combined together with the Access Gate Way. User Equipment (UE) is connected through Air Interface to eNodeB. S1-U interface is used to serve data traffic between eNode-B and S1- MME is signaling-only interface between the eNodeB and the MME [10].

\subsection{Requirements and performance goals for LTE}

The 3GPP Release-8, LTE technology can be used for number of internet applications which includes audio-video streaming, VoIP, internet browsing, real time video streaming, interactive video streaming, internet gaming etc. Because of these heavy applications LTE is being designed to be have a high data rate and very low latency system as indicated by the performance requirement shown in Table 2. There are different frequency spectrum for both uplink and downlink. The maximum bandwidth spectrum is $20 \mathrm{MHz}$ for a UE for both upload and download. This bandwidth flexibility allows the service provider of LTE to deploy the LTE cells with use of any of the bandwidths listed in the Table 2 . This gives flexibility to the service providers to tailor their offering depending on the amount of available spectrum or the ability to start with limited spectrum for lower upfront cost and grow the spectrum for extra capacity [2].

\subsection{OFDM (Orthogonal Frequency Division Multiplex)}

In LTE, both downlink and uplink uses different transmission schemes due to different considerations. In the downlink, a scalable OFDM transmission/multi access technique is used that allows for high spectrum efficiency by utilizing multiuser diversity in a frequency selective channel. On the other hand, a scalable SCFDMA transmission/multi-access technique is used in the uplink as it reduces the peak-to-average power ratio (PAPR) of the transmitted signal [13].

OFDMA allows for flexibility in the transmission bandwidth. LTE is currently specified for bandwidths of 1.4, 3, 5, 10, 15 and $20 \mathrm{MHz}$ In release 10 aggregating multiple carriers are possible in order to increase the desired bandwidth. SCFDMA is at the user side because SC- FDMA's peak to average power ratio which is small. Yet, the more constant power enables high RF power amplifier efficiency in the mobile handsets - an important factor for battery power equipment [4].

Table 2. LTE Performance Metric

\begin{tabular}{|c|c|}
\hline Metric & Requirements \\
\hline Peak Data Rate & DL: 100 Mbps UL: 50 Mbps \\
\hline Mobility Support & $\begin{array}{c}\text { Upto 500 Mph, Optimized for 0-15 } \\
\text { Mph }\end{array}$ \\
\hline Control Plane Latency & $<100 \mathrm{~ms}$ \\
\hline Coverage ( Cell Size ) & $\begin{array}{c}5-100 \mathrm{Km}, \text { degradation after 30 } \\
\text { Km }\end{array}$ \\
\hline Spectrum Flexibility & $1.25,2.5,5,10,15$ and $20 \mathrm{MHz}$ \\
\hline
\end{tabular}

\section{NETWORK MODEL AND IMPLEMENTATION}

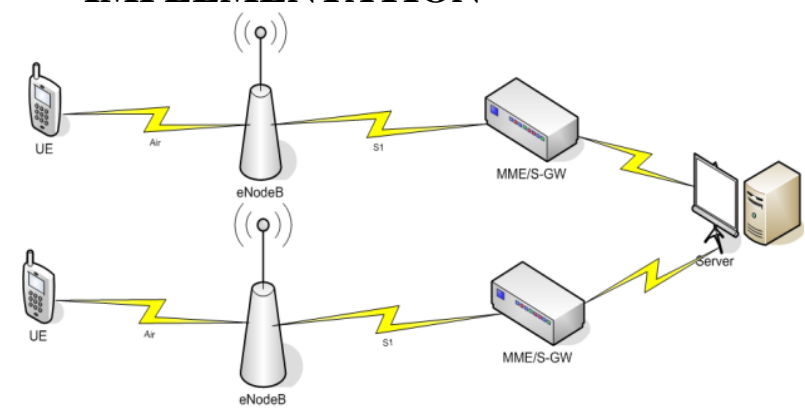

Fig 1: Network Architecture.

This paper simulates one cell of LTE network. Therefore, the part of network needed for simulation is as illustrated in LTE network in Figure 1.

The network model needs to be configured on demand therefore; number of elements and bandwidth parameters between them can be changed, added and/or eliminated. The only limitation to the model that is the eNodeB and Gateway which are permanent and fixed and cannot be moved or multiplied.

The model has five elements namely number (quantity) of User Equipment, at least $1 \mathrm{eNodeB}$, or the base station of the model, which provides the network with the needed flow control data; an Access Gateway to help the network with the flow control and caching Hypertext Transfer Protocol (HTTP) and; one main server providing signaling services. In the network model, LTEQueue, ULAirQueue, DLAirQueue, ULS1Queue and DLS1Queue are implemented to simulate the air interface and S1 interface [5].

\section{PERFORMANCE METRICS}

For evaluating the performance of any system, it should be evaluated on certain criteria; these criteria then decide the basis of performance of any system. Such parameters are known as performance metrics [6] [7]. The three types of performance metrics used to evaluate performance of $\mathrm{UDP} / \mathrm{CBR}$ in this paper are described below:

The parameters which are taken into consideration are end to end average delay, average jitter and average throughput.

Average End to End Delay The delay of a packet in a network is the time it takes the packet to reach the destination after it leaves the source. The average packet delay of a network is obtained by averaging over all transmitted packets in the network. [8]. 
Average Delay $=\sum($ Arrival_Time - Send_Time $) /$

\section{$\sum$ (Arrival_Time - Send_Time $)$}

Jitter It is a term that refers to the variance in the arrival rate of packets from the same data flow, and abnormal jitter values can negatively impact real-time applications like VoIP and video [9].

Average Throughput As defined in the usual way, the time average of the number of bits that can be transmitted by each node to its destination is called the per-node throughput. The sum of per-node throughput over all the nodes in a network is called the throughput of the network [10].

\section{Average Throughput $=\sum P_{R} /\left(\sum t_{s}-\sum t_{e}\right)$}

Where, $P_{R}$ : Received Packet Size, $t_{s}:$ Start Time, $t_{e}:$ Stop Time, Unit: Kbps (Kilo bits per second).

\section{RESULTS}

There are several applications that require reliable data transmission e.g. HyperText Transfer Protocol (HTTP) or Web File Transfer Protocol (FTP), email, Telnet. There are some applications which can tolerate packet loses, however, those are delay sensitive, e.g. multimedia streaming. Alternatively, if someone is watching a movie over the Internet, the packet losses will degrade the quality. If losses are not too much, the application will run. However, if there are large packet delays, there will be several glitches, which are not acceptable.

UDP can be used when an app cares more about "real-time" data instead of exact data replication. For example, VOIP can use UDP and the app will worry about re-ordering packets, but in the end VOIP doesn't need every single packet, but more importantly needs a continuous flow of many of them. Maybe you here a "glitch" in the voice quality, but the main purpose is that you get the message and not that it is recreated perfectly on the other side.

The applications which are delay sensitive require fast transport layer services. Often User Datagram Protocol (UDP) is recommended for these applications instead of Transmission Control Protocol (TCP). The reason is that UDP provides faster transport services as compared to TCP [11].

UDP takes messages from application layer and attaches source and destination port, adds two other fields of error checking and length information, and passes the resulting packet to the network layer [11]. The network layer encapsulates the UDP packet into IP datagram and then delivers the encapsulated packet at receiver end. When a UDP packet arrives at the receiving host, it is delivered to the receiving UDP agent, identified by the destination port field in the packet header.

First, the simulation is carried out for LTE module of ns-2 using UDP/CBR (Constant Bit Rate) traffic and results are obtained. It uses OFDM (Orthogonal frequency division multiplexing) as propagation model. Simulation is performed for both Uplink as well as Downlink performance of LTE. Only results of downlink simulations are shown i.e. the traffic is transmitted from server to the UEs.

\subsection{Impact of Varying Bandwidth}

In this section, we presented the simulation results to evaluate the impact of bandwidth between the eNodeB and the Access Gateway in downlink. Simulation is done for 5, 10,15,20,25 and 30 UEs.

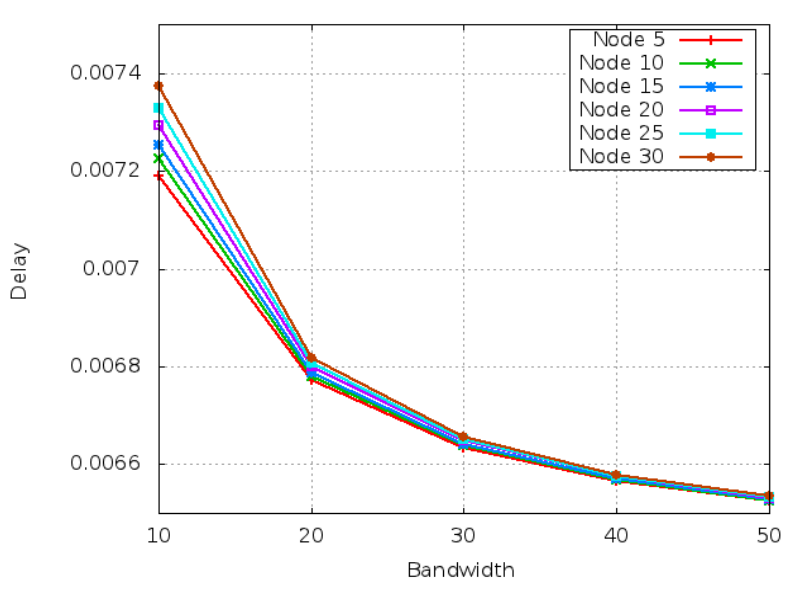

Fig 2. Average delay (Sec) versus bandwidth (Mb) (128Kbps).

Figure 2 and Figure 3 shows average delay as a function of bandwidth. For Figure 2, simulation is done at data rate of 128 Kbps and for Figure 3; simulation is done at data rate of 3 Mbps. We observe that delay decreases with increase in the bandwidth; the reason is this that with increase in the bandwidth, the same traffic has more bandwidth to transfer and transmission delay decreases. In Figure 3, we observe that, delay is higher at higher data rate, it is because, at higher data rate queuing delay and transmission delay is higher [12], if we considering 30 nodes, then they need $90 \mathrm{Mb}$ of link bandwidth, but in our case we have bandwidth of up to 50 $\mathrm{Mb}$, so queuing and transmission delay increases, and average delay becomes very high. As we can see that at higher the number of nodes, higher is the average delay.

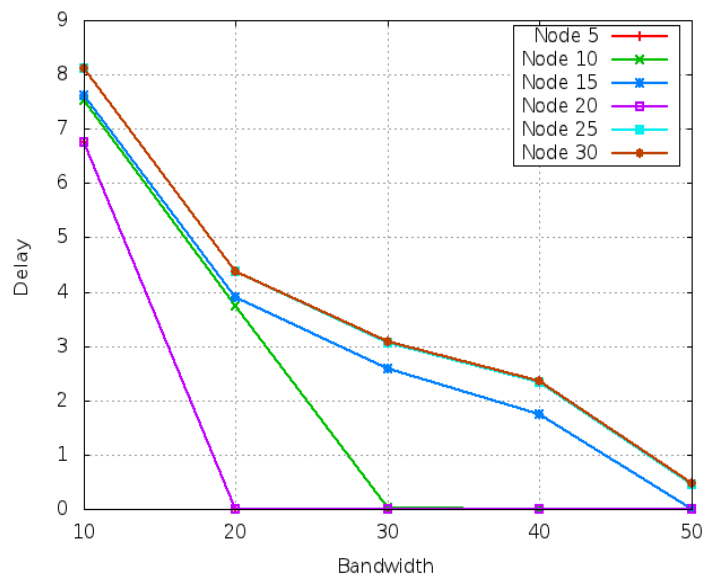

Fig 3.Average delay (Sec) versus bandwidth (Mb) (3Mbps).

At a particular bandwidth, if number of nodes increases, at higher data rate, as we can see, clearly, higher is the average delay, reason is this that with higher data rate, queuing delay increases, so average delay is higher in case of higher data rate. We also observed that, when number of nodes changes from 30 to 5 nodes at a particular bandwidth, at data rate of $128 \mathrm{kbps}$, average delay decreases maximum up to $0.13 \%$, and, when number of nodes changes from 30 to 5 nodes, at data rate of $3 \mathrm{Mbps}$, average delay decreases maximum up to $98 \%$. 


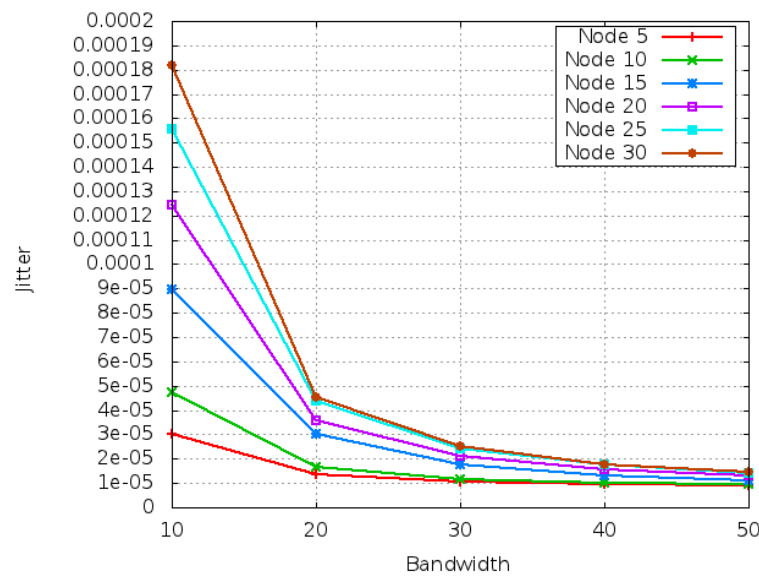

Fig 4. Average jitter (Sec) versus bandwidth (Mb) (128Kbps).

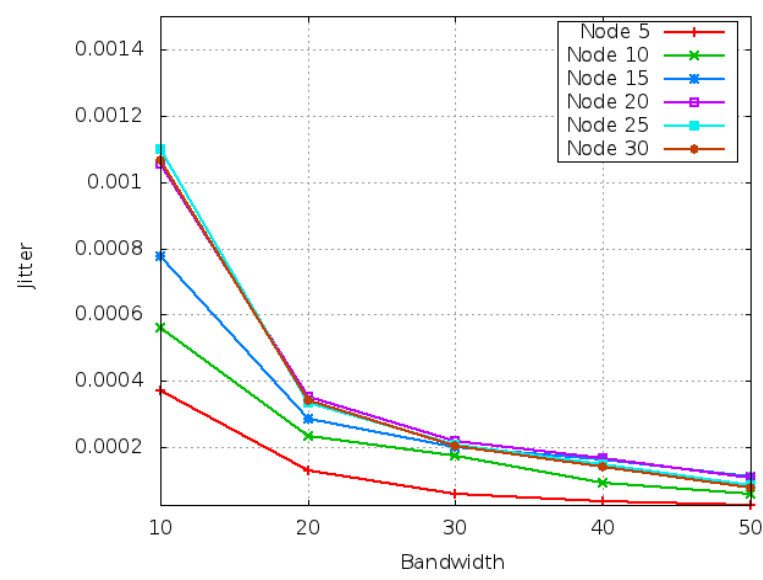

Fig 5. Average jitter (Sec) versus bandwidth (Mb) (3Mbps).

Figure 4 and Figure 5 shows average jitter as a function of bandwidth. We have observed that average Jitter decreases with increase in bandwidth. And we also observed that, higher the data rate, higher is the average jitter. From Figure 4 and Figure 5, we can see that the data rate $128 \mathrm{Kbps}$ produces lower average jitter than that of data rate of $3 \mathrm{Mbps}$.

Figure 6 and Figure 7 shows average throughput as a function of bandwidth. We have observed that for data rate of 128 Kbps, average throughput is almost constant at all bandwidths, for data rate of $3 \mathrm{Mbps}$, average throughput initially increases for lower bandwidths but after increasing the bandwidth average throughput becomes almost constant. Because for CBR traffic by default we have $128 \mathrm{Kbps}$ of data rate, so here we have enough of bandwidth, so it is easily getting its required bandwidth, so average throughput remains constant. In case of $3 \mathrm{mbps}$, e.g. if we have 5 nodes, and data rate is $3 \mathrm{Mbps}$, and the minimum required bandwidth for which sender can send its traffic with maximum average throughput is $15 \mathrm{Mb}$, so up to $15 \mathrm{Mb}$ bandwidth, average throughput will increase in case of 5 nodes and after that it will be constant. We also observed that, when number of nodes changes from 30 to 5 nodes at a particular bandwidth, at data rate of $128 \mathrm{Kbps}$, average throughput decreases maximum up to $0.25 \%$, and, when number of nodes changes from 5 to 30 nodes, at data rate of $3 \mathrm{Mbps}$, average throughput decreases maximum up to $44 \%$.

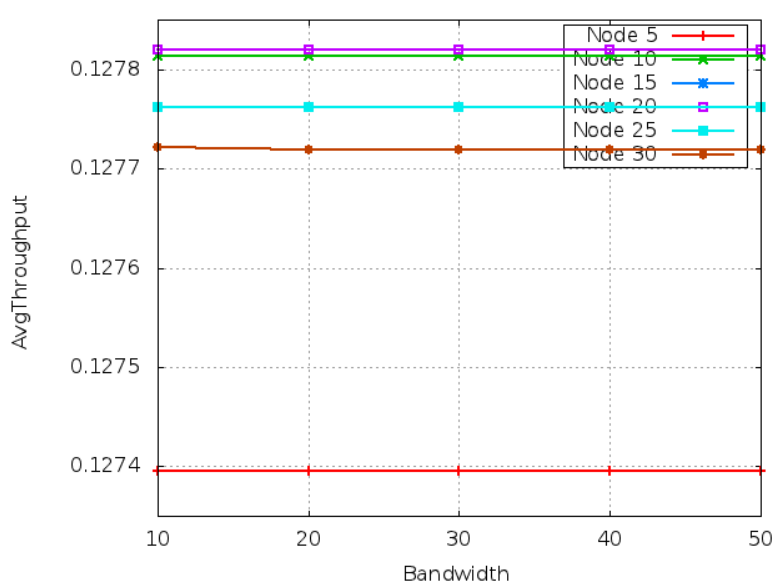

Fig 6. Average throughput (Mbps) versus bandwidth (Mb) (128 Kbps).

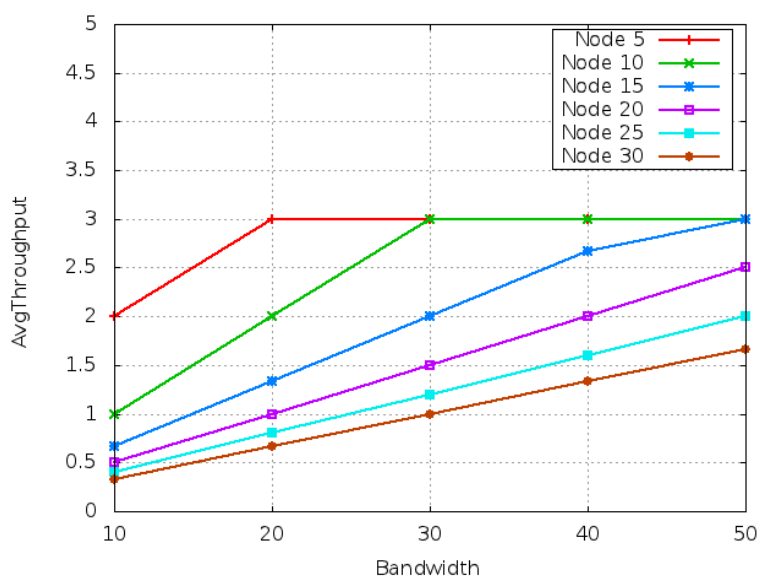

Fig 7. Average throughput (Mbps) versus bandwidth (Mb) (3 Mbps).

\subsection{Impact of Varying Number of Subscribers}

In this section, we present the simulation result of impact of the number of subscriber station on the performance of UDP traffic. Numbers of UEs are varied from 1 to 226 and simulation is done for the bandwidth of $20 \mathrm{Mb}$ and $40 \mathrm{Mb}$ between eNodeB and Access Gateway. Simulation is done for CBR. This simulation is done in downlink traffic i.e. the traffic is transmitted from server to the UEs.

Figure 8 shows average delay as a function of number of nodes. We have observed average delay remains constant up to 150 nodes and increases sharply after 150 ,the reason is that in case of CBR, rate is $128 \mathrm{Kbps}$ and available bandwidth is 20 $\mathrm{Mb}$, so up to 150 nodes, all nodes are getting their required bandwidth (i.e. $150 * 128 \mathrm{~Kb}=20 \mathrm{Mb}$ ). But after 150 nodes due to increase in transmission delay overall delay increases. For $40 \mathrm{Mb}$ bandwidth, till 225 nodes, all the nodes are getting their required bandwidth properly (i.e. $225 * 128 \mathrm{~Kb}=29 \mathrm{Mb}$ ), so average delay is very less, but if we will increase number of nodes beyond 225 , then the behavior of $40 \mathrm{Mb}$ line will be same as $20 \mathrm{Mb}$ line. We also observed that, when bandwidth decreases from $40 \mathrm{Mb}$ to $20 \mathrm{Mb}$ at a particular node, average delay decreases maximum up to $99 \%$. 


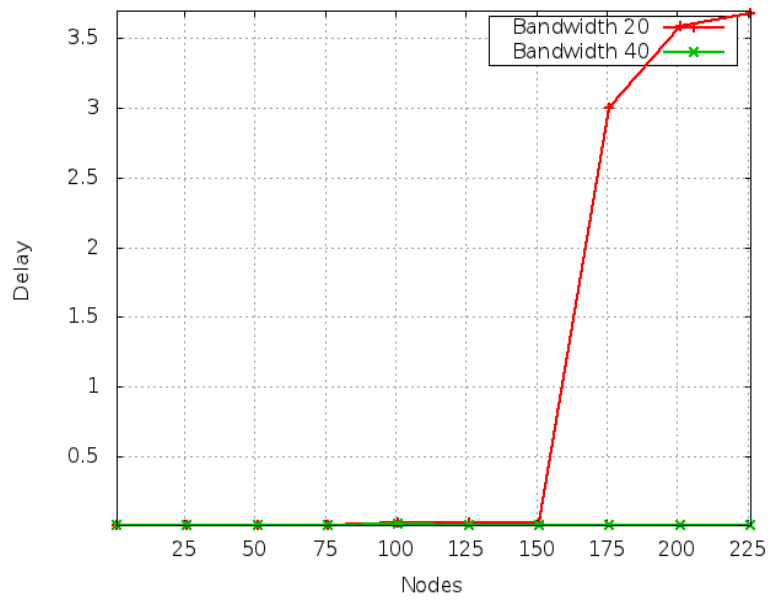

Fig 8. Average delay (Sec) versus number of subscriber.

Figure 9 shows average jitter as a function of number of nodes. We have observed that average jitter increases with increasing the number of nodes. We also observed that average jitter is higher for lower bandwidth.

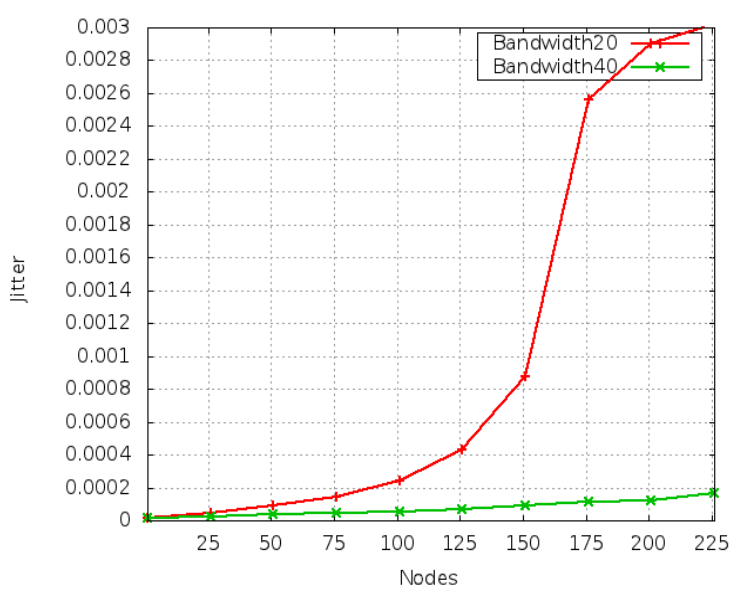

Fig 9. Average jitter (Sec) versus number of subscriber.

Figure 10 shows average throughput as a function of number of nodes. For $20 \mathrm{Mb}$ bandwidth, average throughput remains almost constant up to 150 nodes, it is around $128 \mathrm{Kbps}$, because CBR traffic data rate is $128 \mathrm{Kbps}$, and after 150 nodes average throughput decreases rapidly, because all the nodes cannot send at required rate of $128 \mathrm{Kbps}$, so average throughput decreases very sharply. For bandwidth of $40 \mathrm{Mb}$, average throughput remains constant up to 225 nodes, because in this case each node is getting its required bandwidth and throughput remains constant, and it might be possible that after increasing the number of nodes beyond 225, its average throughput might also decrease rapidly. We know that Wireless is a "shared medium," which means that the clients in an area are sharing bandwidth. The bandwidth resources are finite, so as you increase the number of clients in an area, the network becomes slower for all clients. We also observed that, when bandwidth decreases from $40 \mathrm{Mb}$ to $20 \mathrm{Mb}$ at a particular node, average throughput decreases maximum up to $31 \%$. It is because that, when bandwidth increases, it causes decrease in transmission delay and average delay decreases with increase in bandwidth.

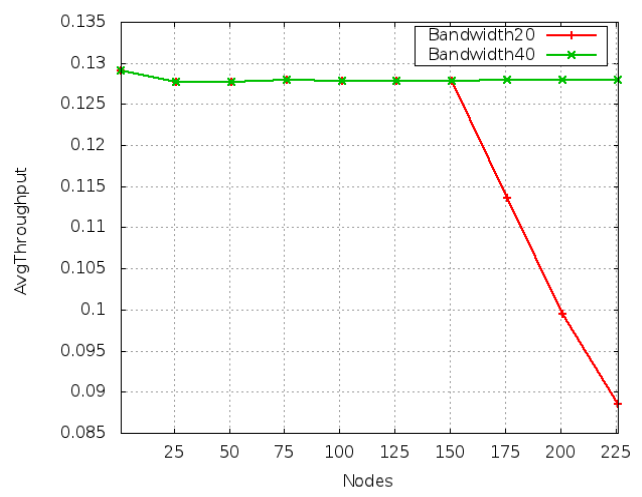

Fig 10. Average throughput $(\mathrm{Mb})$ versus number of subscriber.

\subsection{Impact of Varying Packet Size}

In this section, we present simulation results to evaluate the impact of different packet size over UDP traffic. Packet size is varied from 100 bytes to 1000 bytes. Simulation is done on two bandwidth i.e. $20 \mathrm{Mb}$ and $40 \mathrm{Mb}$. Simulation is done on single UE. UDP traffic includes CBR traffic. This simulation is done in downlink i.e. the traffic is transmitted from server to the UEs.

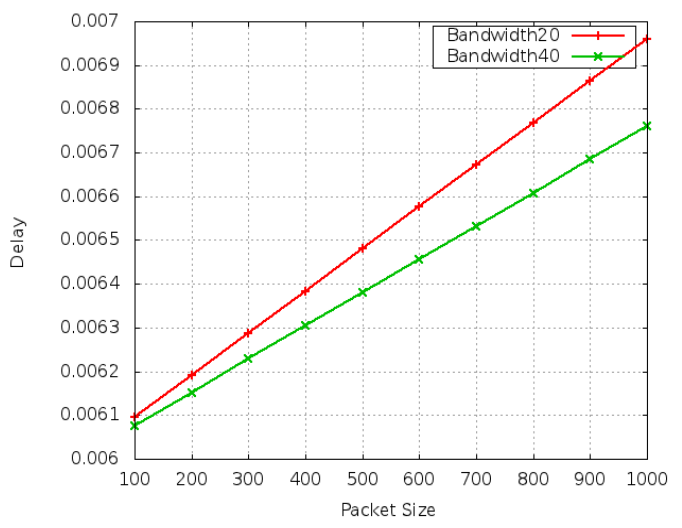

Fig 11. Average delay (Sec) versus packet size (Bytes).

Figure 11 shows average delay as a function of packet size. We have observed that average delay increases with increase in packet size, reason is this, that, increase packet size, then transmission delay increases and packet takes more time to transmit into the link, so average delay increases. We also observed that average delay is higher in case of bandwidth of $20 \mathrm{Mb}$ as compared to bandwidth of $40 \mathrm{Mb}$, reason is that if bandwidth is higher for a particular packet size, then transmission delays decreases and but queuing delay remains same, so overall average delay decreases. We also observed that, as the band width increases from $20 \mathrm{Mb}$ to $40 \mathrm{Mb}$ for a particular packet size, average delay decreases very little by only $2.9 \%$.

Figure 12 shows average jitter as a function of packet size. We observe that average jitter does not follow any specific pattern, but it shows that lower the bandwidth, higher is the jitter.

Figure 13 shows average throughput as a function of packet size. We have observed that, average throughput almost remains around $128 \mathrm{Kbps}$, this because, with increase in packet size, CBR traffic rate is $128 \mathrm{Kbps}$, but we have enough of bandwidth, so average throughput remains around 128 Kbps. We also observed that average throughput is same for 
both $20 \mathrm{Mb}$ and $40 \mathrm{Mb}$ of bandwidths. Reason is this that if bandwidth is higher for a particular packet size, then also it needs only $128 \mathrm{~Kb}$ of bandwidth to transmit, so average throughput remains same. We also observed that, as the band width increases from $20 \mathrm{Mb}$ to $40 \mathrm{Mb}$ for a particular packet size, average throughput remains same.

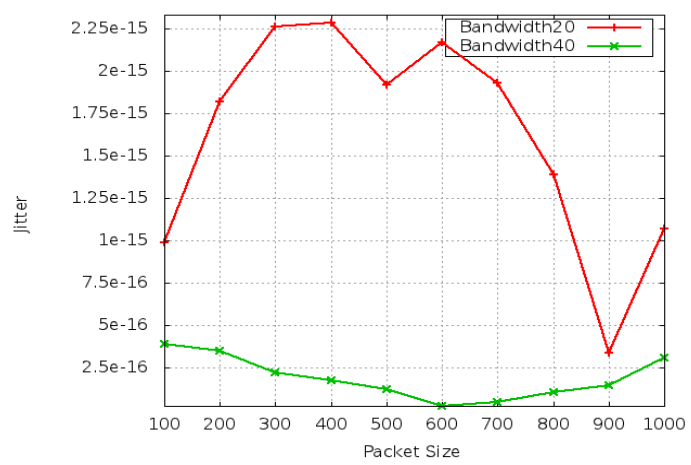

Fig 12. Average jitter (Sec) versus packet size (Bytes).

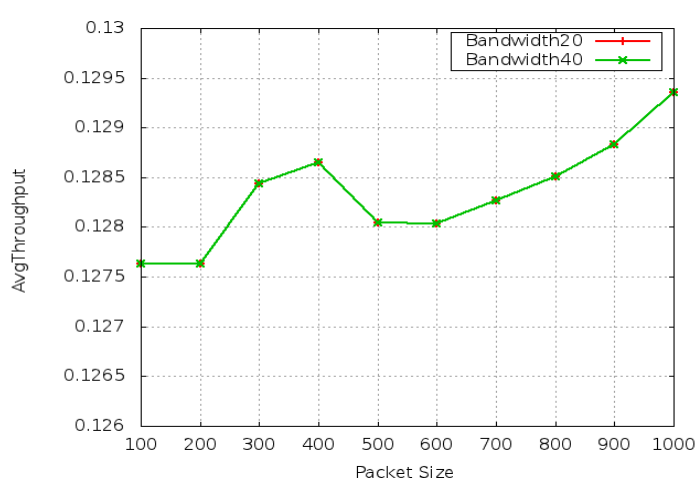

Fig 13. Average throughput $(\mathrm{Mb})$ versus packet size (Bytes).

\section{SUMMARY AND CONCLUSION}

In this paper, we evaluated the performance of LTE network under the different types of traffics.

We evaluated the performance of UDP/CBR over LTE network access in downlink channels. We carried out simulations using Network Simulator (NS2). While evaluating the performance, we focused on the following parameters: (I) Average Delay, (II) Average Jitter, and (III) Average Throughput. Effect of bandwidth, number of subscriber and packet size is evaluated. Results show that as the bandwidth increases the average delay decreases and average jitter also decreases, but average throughput increases. With increase in the number of subscribers, average delay and average jitter increases and average throughput decreases. And in case of packet size; average delay, average jitter and average throughput increases.

There are many directions for future work e.g. one may propose an analytical model for the performance of LTE. Further, one may devise one's own scheduling technique and may evaluate its impact on the performance of LTE.

\section{REFERENCES}

[1] Arunabha Ghosh, Jun Zhang, Jeffrey G. Andrews and Rias Muhamed, "Fundamentals of LTE", Prentice Hall, September 10, 2010.

[2] LTE White Paper by Verizon, "LTE: The Future of Mobile Broadband Technology", Available at http://lteworld.org/whitepaper/lte-futuremobilebroadband-technology.

[3] 3G LTE tutorial, "3G LTE Tutorials - 3GPP Long Term Evolution", viewed on 03.08.15, Available at http://www.radioelectronics.com/info/cellulartelecomms/ lte-long-termevolution/3g-lte-basics.php.

[4] Harri Holma and Antti Toskala, "LTE for UMTS OFDMA and SCFDMA Based Radio Access", Nokia Siemens Networks, Finland.

[5] Iana Siomina and Stefan W"anstedt, "The Impact of QoS Support on the End User Satisfaction in LTE Networks with Mixed Traffic", IEEE 19th International Symposium on Personal, Indoor and Mobile Radio Communications, 2008. PIMRC 2008.

[6] Bijan Paul, Md. Ibrahim, Md. Abu Naser Bikas, "Experimental Analysis of AODV \& DSR over TCP \& CBR Connections with Varying Speed and Node Density in VANET", International Journal of Computer Applications, Vol. 24, No. 4, June 2011.

[7] Ritika Sharma, Kamlesh Gupta, "Comparison based Performance Analysis of UDP/CBR and TCP/FTP Traffic under AODV Routing Protocol in MANET", International Journal of Computer Applications, Volume 56- No.15, October 2012.

[8] Pan Li, Yuguang Fang, Jie Li, "Throughput, Delay, and Mobility in Wireless Ad Hoc Networks", In Proceeding of IEEE INFOCOM, San Diego, CA ,14-19 March 2010.

[9] D. Zhou, W. Song, N. Baldo, M. Miozzo, "Evaluation of TCP Performance with LTE Downlink Schedulers in a Vehicular Environment", Proc. of 9th IEEE International Wireless Communication and Mobile Computing Conference (IWCMC), pp. 1064-1069, July 2013.

[10] S. Omar, A. Ancora, D.T.M. Slock, "Performance Analysis of General Pilot-Aided Linear Channel Estimation in LTE OFDMA Systems with Application to Simplified MMSE Schemes", Proc. of 19th IEEE International Symposium on Personal, Indoor and Mobile Radio Communications(PIMRC), pp. 1-6, September 2008.

[11] J.J. Sanchez, D.M. Jimenez, G. Gomez, J.T. Embrambasaguas, "Physical Layer Performance of Long Term Evolution Cellular Technology", Proc. of 16th IEEE Mobile and Wireless Communications Summit (IST), pp. 1-5, July 2007.

[12] Y. Li, X. Chen, W. Zhao, B. Cao, "Packet Scheduling with QoS Supporting LTE Downlink MIMO System", Proc. of $1 \mathrm{st}$ IEEE International Conference on Communications in China (ICCC), pp. 97-102, August 2012.

[13] X. Li, W. Bigos, D. Dulas, Y. Chen, U. Toseef, C. Goerg, A. Timm-Giel, and A. Klug, " Dimensioning of the LTE Access Network for the Transport Network Delay QoS ", Proc. Of 73rd IEEE Vehicular Technology Conference (VTC), pp. 1-7, May 2011. 